\title{
Ischaemic contracture of the hand
}

\section{G. Balakrishnan}

Right Hospitals, Kilpauk, Chennai - 600 010, Formerly Professor and Head, Institute for Research and Rehabilitation of Hand and Department of Plastic Surgery, Stanley Medical College and Govt. Stanley Hospital, Chennai - 600 001, India

Address for correspondence: G. Balakrishnan, Right Hospitals, Kilpauk, Chennai - 600 010, India. E-mail: righthospitals@yahoo.co.in

\section{INTRODUCTION}

schaemic contracture of the hand is a complex, variable deformity occurring as a sequelae following injury and ischaemic damage to the forearm muscles due to neglected compartment syndrome of various causes. This affects the muscles primarily and the nerves secondarily. The management of such a problem is a challenging proposition to hand surgeons.

Ischaemic contracture of the hand is defined as sequelae of ischaemic injury to the muscle and nerves of the limb. Identifying and releasing the increased intracompartment pressure before the irreversible ischaemic damage sets in can best prevent this problem.

Ischaemic contracture may affect a single finger or the entire forearm flexor muscles.

\section{HISTORY}

A century ago, Richard Von Volkmann of Halle, Germany (1881) identified that posttraumatic contractures of the hand occurred as sequelae to ischaemia. ${ }^{[1]}$ Before this, it was attributed to nerve injuries. Murphy, in 1914, attributed the same to internal pressure and venous obstruction and advocated splitting the fascia of forearm from the elbow, distally. After World War I, interest switched to arterial trauma, when Griffith in 1940 attributed it to reflex spasm of collateral circulation due to arterial injury.

In 1975, Holden suggested that the "compartment syndrome" was an acute ischaemic condition, which may or may not be associated with arterial injury and if unresolved, in time, would result in residual contractures. ${ }^{[2]}$ Later, Whitesides, Mubarak and Masten introduced the various techniques for measuring the compartment syndrome. ${ }^{[3]}$

Ischaemic contracture of hand may be dealt with under two headings.

1. Ischeamic contracture of the forearm described by Volkmann in 1881.

2. Ischaemic contracture of the intrinsic muscles of the hand described by Ricardo Finochietto in $1920^{[4]}$ who originally described ischaemic contracture of the intrinsic muscles of the hand as Volkmann's retraction of the intrinsic muscles of the hand.

\section{Pathogenesis}

The key factors in all cases of ischaemic contracture is the decrease in the compartment size or increase in the content in a closed unyielding osteofascial compartment, which is sufficient to cause occlusion of small vessels. The occlusion quickly leads to muscle and nerve ischaemia. If the compartment pressure is unrelieved in time, it results in muscular damage with contracture and nerve damage.

Decrease in compartment size could occur due to many causes including, for example, constrictive casts and dressings, sustained localised external pressure, thermal injuries, burn eschar or closure of fascial defects. On the other hand, an increase in compartment content could be due to hemorrhage, vascular injury, bleeding 
diathesis or anticoagulation. Similarly, an increased volume of the compartment could occur due to soft tissue or skeletal trauma, post ischaemic swelling, infection, intra arterial or intravenous injection, infiltrated infusion, exercise, surgical intervention or compression and immobilization.

The tolerance of muscles and nerves to ischaemia varies in terms of time and compartment pressure. Nerve ischaemia produces altered sensations within 30 minutes but irreversible ischaemic damage begins after 12 hours. Muscle ischaemia produces functional changes in the first four hours and irreversible damage sets in 6 to 12 later, and skin changes are seen after 12 hours.

The normal pressure in the tissue compartment is 0 to 8 $\mathrm{mm}$. Hg. It normally rises with muscle contraction and drops to 0 with relaxation. A resting compartment pressure of over $30 \mathrm{~mm} \mathrm{Hg}$ above the diastolic pressure of the individual obliterates capillary circulation and leads to ischemia. The ischemic cycle is described in Figure 1.

\section{ESTABLISHED VOLKMANN'S ISCHEMIC CONTRACTURE}

\section{Classification}

Ischemic injuries are classified in different ways. Thus Seddon ${ }^{[5]}$ classified ischemia into three types depending on severity of involvement as follows:

Grade I: Ischaemia

Grade II: Ischaemic contracture

Grade III: Ischaemic contracture with nerve involvement On the other hand, Zancolli ${ }^{[6]}$ grouped them into 4 types depending upon the structures involved as given below: Type I: Contracture involving forearm muscles with normal intrinsic muscles

Type II: Contracture involving forearm muscles with paralysis of intrinsic muscles

Type III: Contracture involving forearm muscles with contracture of the intrinsic muscles

Type IV: Combined type

Tsuge $e^{[7]}$ classified ischemic contractures into three types according to the extent, muscle groups involved and presentation into mild, moderate and severe.

\section{Stages of evolution}

The ischaemic contracture is the result of neglected compartment syndrome, establishment of final deformity takes nearly 6 months, during which time a series of changes take place. The different stages of evolution include the acute stage (vascular crisis), sub acute or delayed stage (evolutionary) and the final or established stage. Patients may present at different stages although unfortunately, most often as an established deformity.

When a patient with ischaemic contracture presents, the following investigations may be performed:

1. Direct compartment pressure measurement (manometry)

2. Plain X-rays of arm and forearm to find out the fractures and dislocations

3. Angiogram: In the acute stage, if any major vessel injury is suspected an angiogram is justified. In the established stage, an angiogram may be necessary while planning for free functional muscle transfer.

4. CT scan with contrast has become an essential noninvasive investigation as it assesses the extent and severity of various muscle groups involved, and allows the surgeon to plan to release exactly the muscle involved as well as to estimate the prognosis. It is a complimentary test in grouping patients under the Tsuge classification. An angiogram can be avoided if a contrast CT is taken.

5. An MRI serves the same purpose as a CT though it is more expensive. It provides better soft tissue image resolution and it does not require contrast. It is particularly useful in identifying the course and compression of the nerves.

6. Direct nerve stimulation studies are useful during surgery in confirming the status of the nerve. They also help in choosing between neurolysis or nerve grafting as treatment.

7. Reperfusion injury parameters may be monitored. For example, presence of myoglobin in urine following considerable delay in fasciotomy reflects two problems: that the muscle has already suffered irreversible ischaemic damages and secondly, that care has to be taken to prevent systemic deterioration due to reperfusion injury.

\section{STAGES OF PRESENTATION}

\section{Acute stage}

The acute stage presentation is common in children, in patients with supra-condylar fracture or in those with injury to brachial artery. When such patients present, the following six 'P's are essential to pin-point the 
diagnosis:

i. Pain

ii. Positive passive stretch test

iii. Paresthesia

iv. Paralysis

v. Painful tense forearm

vi. Pulseless limb \pm i.e., may or may not be seen as a sign

Compartment pressure studies are useful only in the acute stage and are advocated when the clinical diagnosis is equivocal or the patient is comatosed.

There are different methods of manometry including the Whitesides Infusion technique, the slit catheter technique of Mubarak, the continuous monitoring technique of Matsen or by using the Stryker STIC device. We use the infusion technique designed by Whitesides [Figure 2]. A $20 \mathrm{ml}$ syringe with a plunger at the $15 \mathrm{ml}$ mark is attached to an appropriate three-way stopcock with two plastic i. v. tubes, one connected to the mercury manometer and one to a sterile 18 gauge needle. The 18 gauge needle is inserted into a bottle of normal saline, and the saline is aspirated into approximately half the length of the plastic tube. The three-way stopcock is turned to close off the tubing so that the saline is not lost during transfer of the needle. The 18-gauge needle is inserted into the compartment in which the tissue pressure is to be measured. The stopcock is then turned so that the syringe is open to both extension tubes. The portion of the tube containing the top of the column of saline is then placed at the same level as the tip of the needle in the patient.

The pressure in the system is then increased by gradually depressing the plunger of the syringe. When the pressure in this system has just surpassed the compartment tissue pressure, a small amount of saline will be injected into the compartment and the saline column in the plastic tube will be seen to be moving. When the column of saline moves, the pressure on the syringe plunger is immediately withdrawn and the level of mercury is read on the gauge. This is the tissue pressure of a specific compartment in mm of mercury. The measurement is always taken twice. If there is a marked difference, a third measurement should be taken using a new 18-gauge needle each time.

Fasciotomy is indicated if the pressure is above $30 \mathrm{~mm}$ of mercury. The common errors in this method are injection of too much saline into the compartment, placing the needle into a tendon rather than a muscle belly and aspirating a tissue plug of muscle into the needle before the tissue pressure is measured.

\section{Treatment}

The goal in treatment is to re-establish the circulation of affected muscles and nerves. In adults immediate surgery is recommended while in children surgery can be delayed by between 24- 48 hours. Children usually withstand ischemia better and hence continuous observation is essential.

Naturally the cause has to be treated. Limb elevation, compartment pressure measurement followed by fasciotomy or delayed wound closure are other options. Fasciotomy is performed when corrective measures like removing the cause like constricting bandages or reduction of fractures does not improve the circulation within 3 to 4 hours. The indications for fasciotomy include:

- Stretch pain

- No sign of clinical improvement

- Increased intra compartment pressure

- Ischaemic changes in finger tips.

Some of the useful points to consider when performing a fasciotomy include taking a long incision to decompress the entire forearm and sparing as many veins as possible. In case two incisions are taken, the forearm skin flap should have a minimum width of $5 \mathrm{cms}$ and should be raised along with deep fascia so that viability of skin flap will be reliable and can cover the exposed median nerve and tendons. The deep fascia over the Profundus muscle must always be laid open and the carpal tunnel should also be decompressed. The non-viable portion of muscle must be excised.

The technique of fasciotomy is illustrated in Figures 3 and 4. An extensive fasciotomy of the involved compartments including both superficial and deep flexor compartments from the flexor crease of elbow to the wrist is required. The Median and Ulnar nerves are released from the muscle arches in the proximal forearm. Individual muscles are palpated and epimysium is opened if in doubt. If the dorsal compartment is also tight, it is also opened. After 10 to 15 minutes of fasciotomy, small vessel bleeding will increase which is controlled and the wound left open. If a major vessel (e.g., brachial artery) injury is present, an attempt should be made to repair the vessel. Finally, an adaptive non-constrictive 
moistened dressing is applied. Delayed skin closure or grafting is done in 3 to 5 days.

Mechanical spasm of the vessel may be overcome by

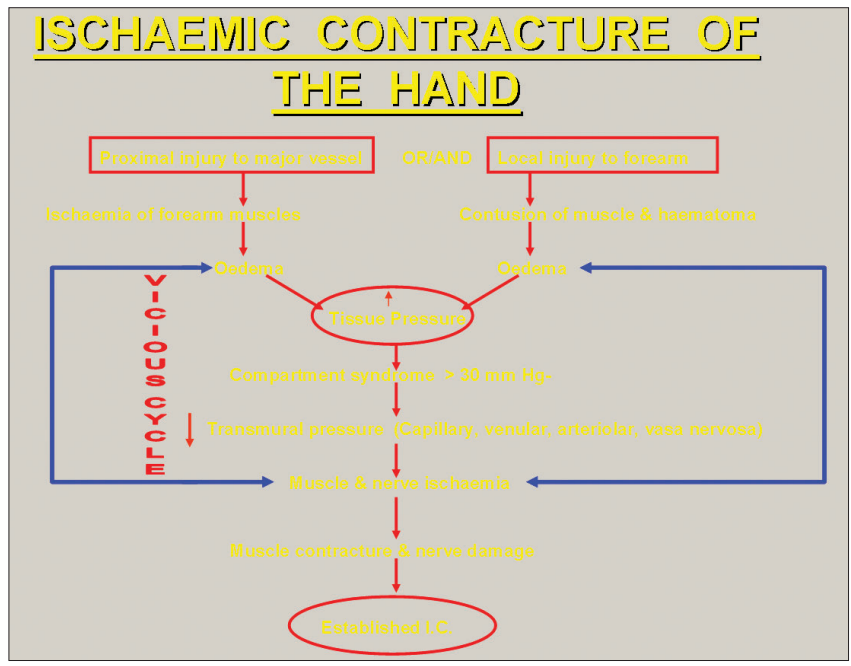

Figure 1: Ischaemic contracture of the hand

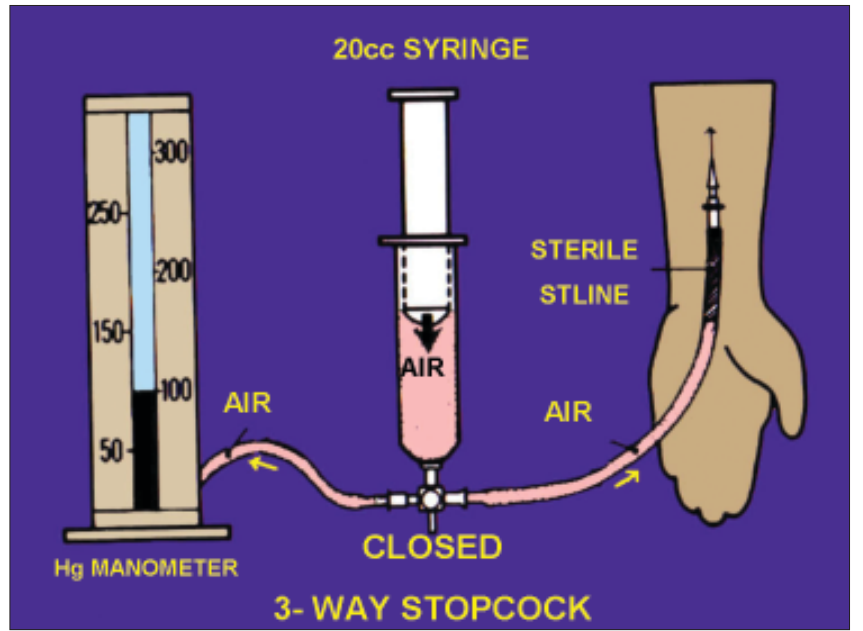

Figure 2: 3-way stopcock

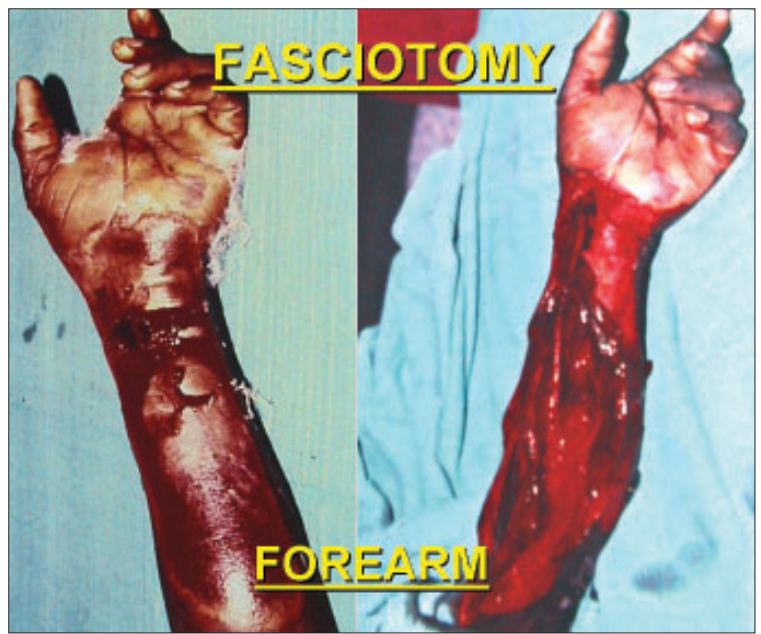

Figure 3: Forearm injecting a bolus of fluid through the narrow segment proximally.

There are some key issues that must be noted to decide

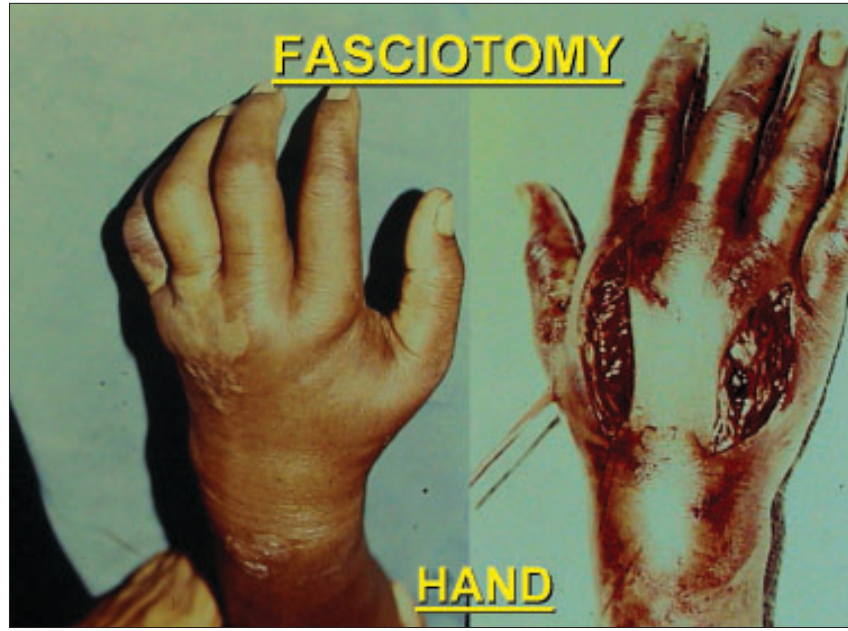

Figure 4: Hand

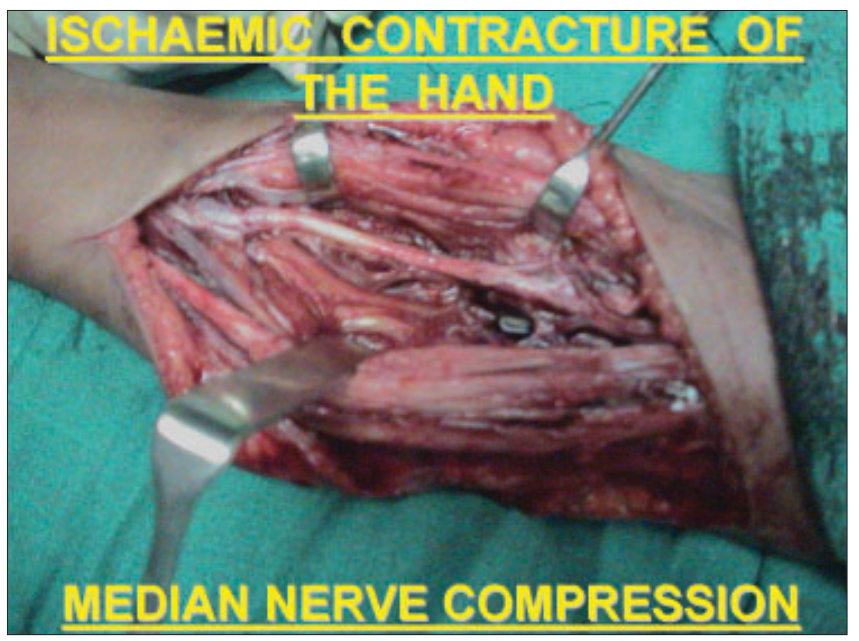

Figure 5: Median nerve compression

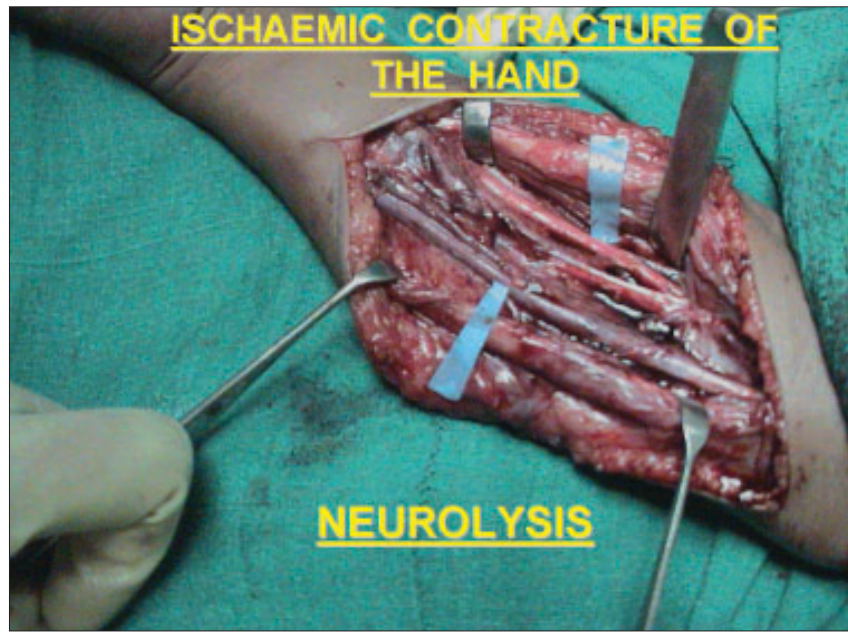

Figure 6: Neurolysis

Indian J Plast Surg January-June 2006 Vol 39 Issue 1 
success of a fasciotomy, including the amount of skin recession at release incisions, viability of muscles by colour, the ability of muscles to contract on pinching,

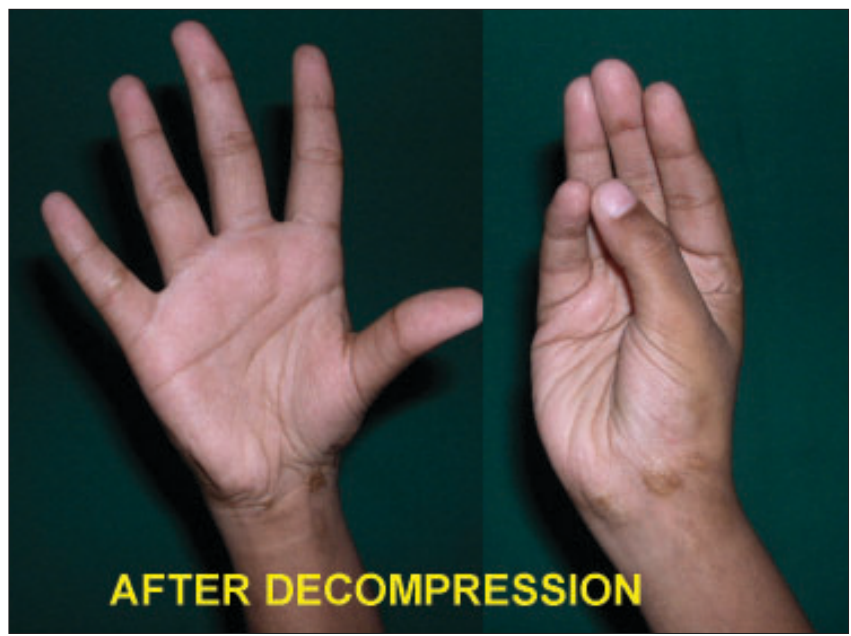

Figure 7: After decompression

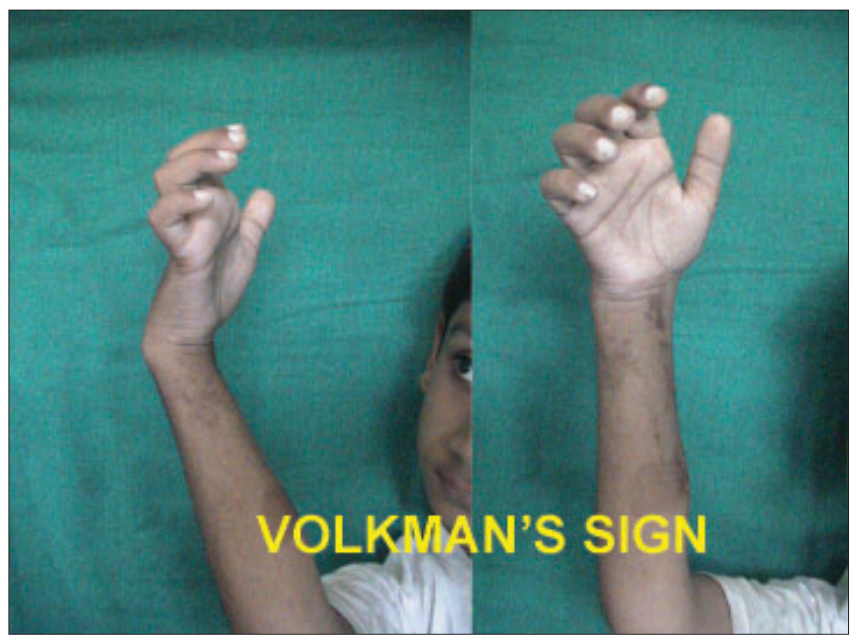

Figure 8: Volkman's sign

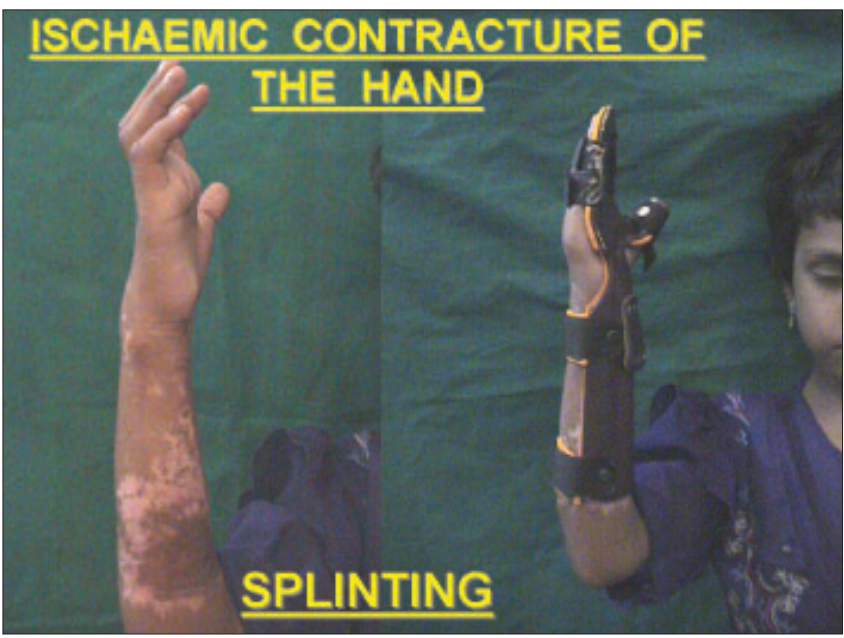

Figure 9: Splinting return of pulsation in the vessels distally, passive stretch of flexors and change of the colour in fingertips to confirm the viability.

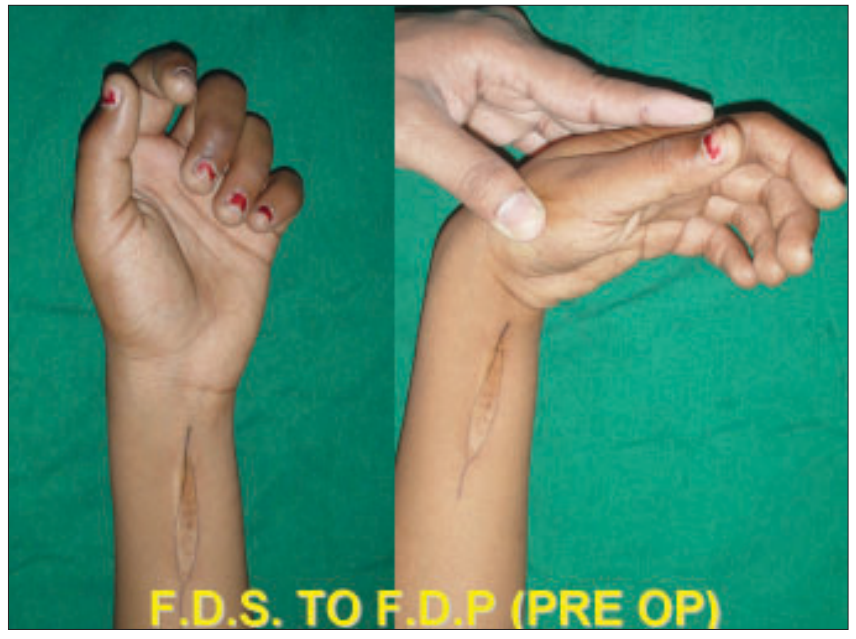

Figure 10: F. D. S. to F. D. P. (pre op)

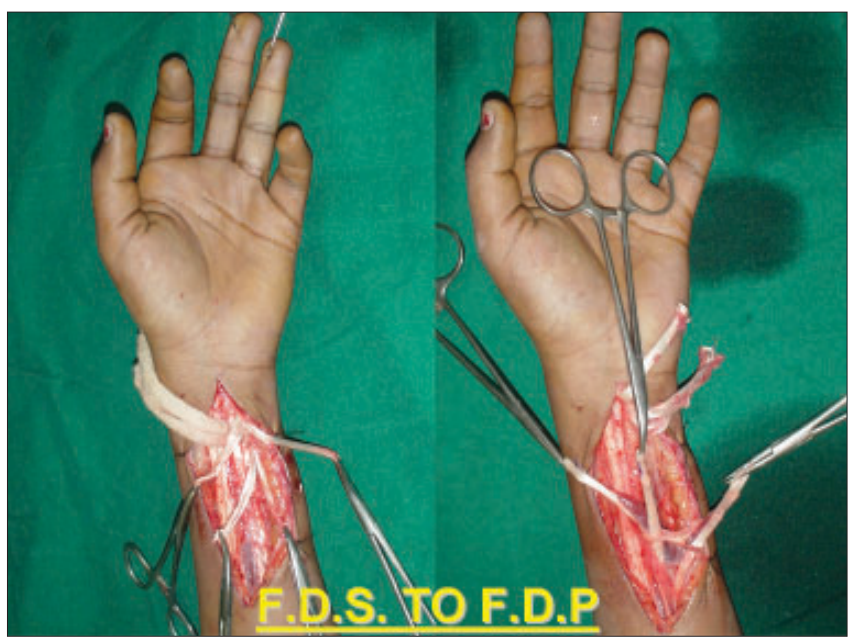

Figure 11: F. D. S. to F. D. P.

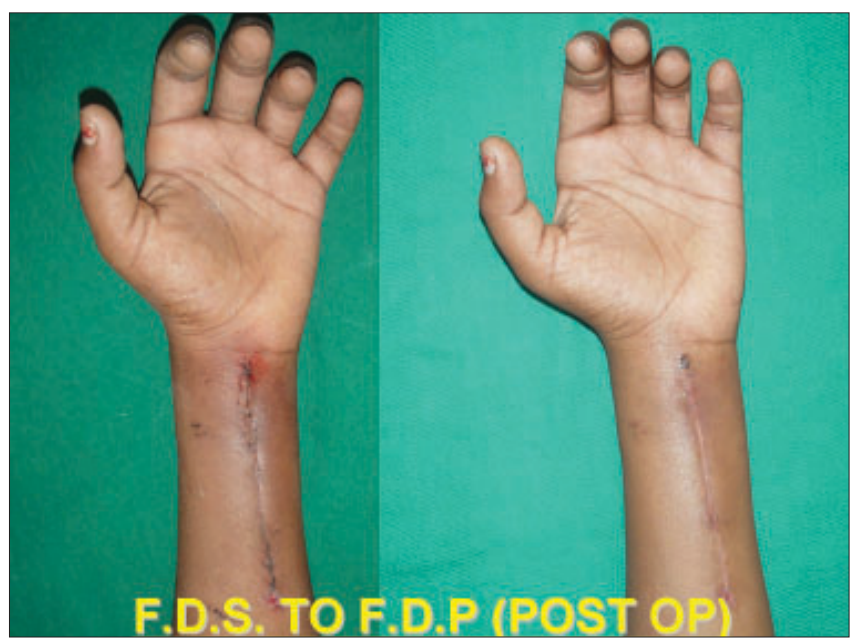

Figure 12: F. D. S. to F. D. P. (post op) 


\section{Sub-acute or delayed (paralytic) stage}

This stage extends from one to two days upto 3 to 6 months when the spontaneous recovery of muscles and

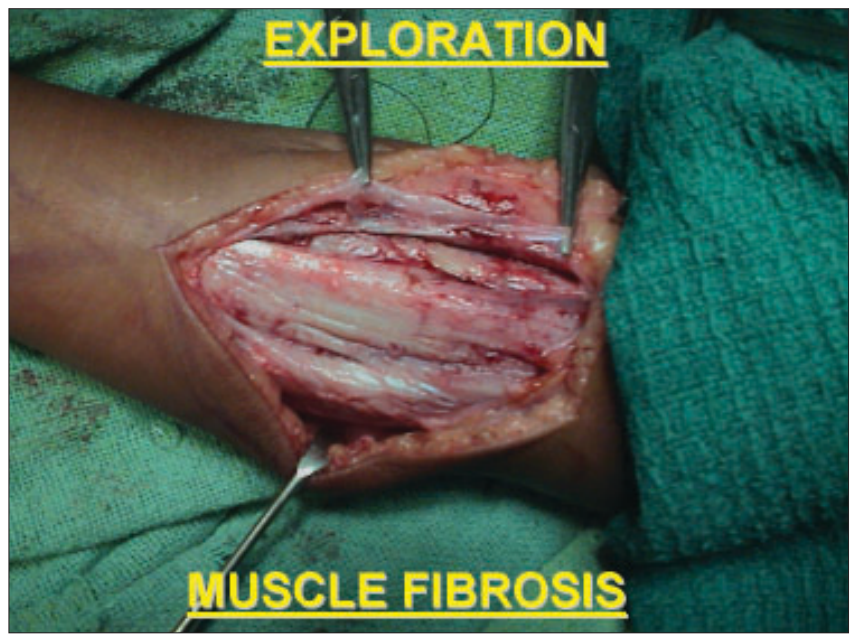

Figure 13: Muscle fibrosis

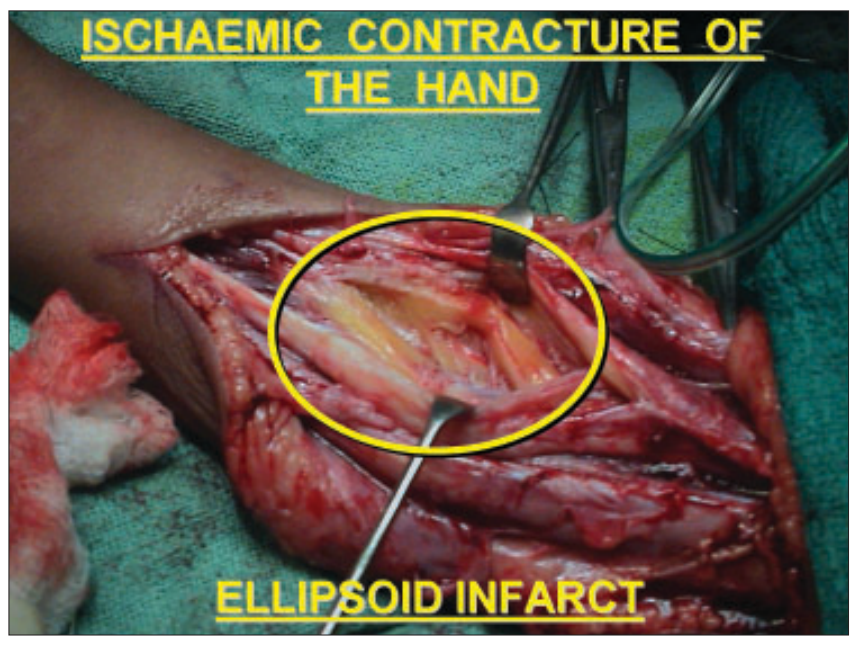

Figure 14: Ellipsoid infarct

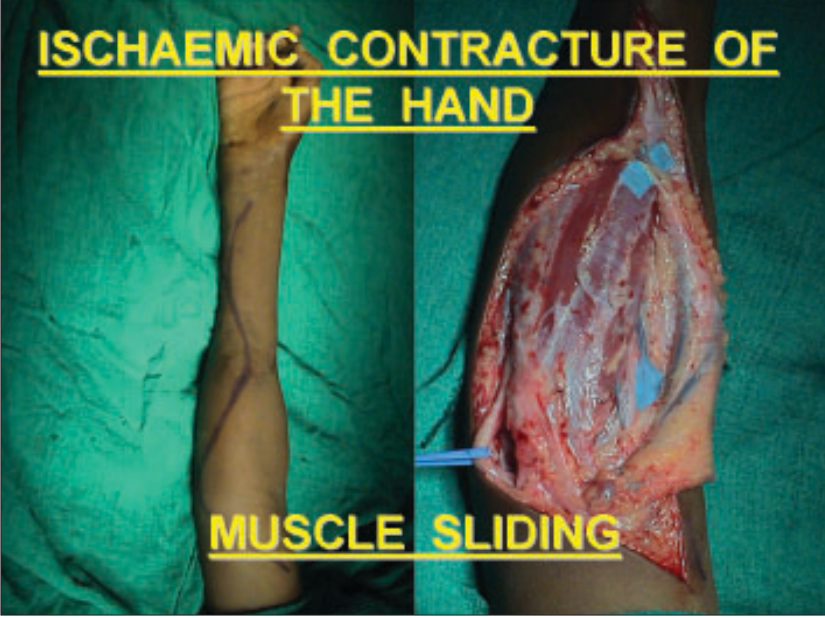

Figure 15: Muscle sliding nerves stops. The patient presents with oedema and induration in the forearm and hand, or paraesthesia or anaesthesia in the hand and fingers. Trophic changes

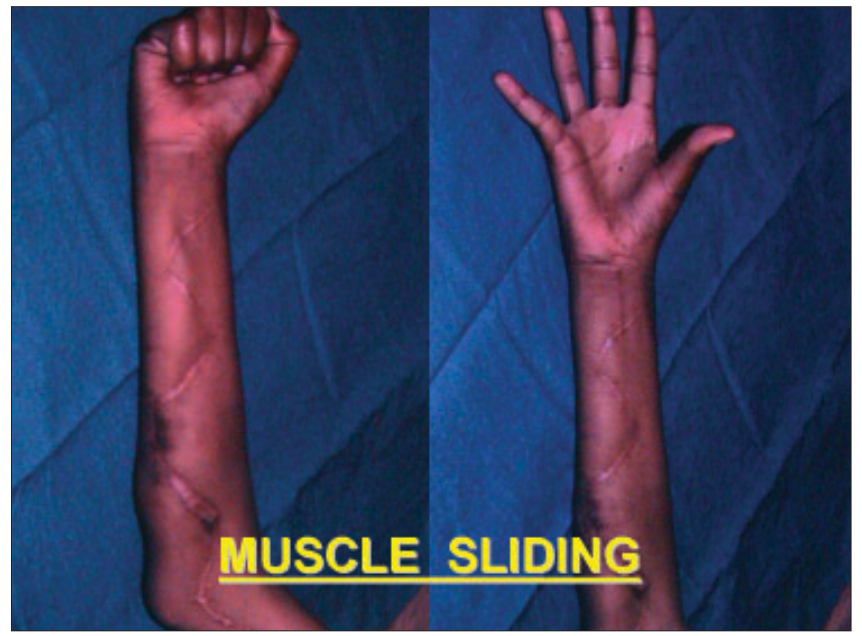

Figure 16: Muscle sliding

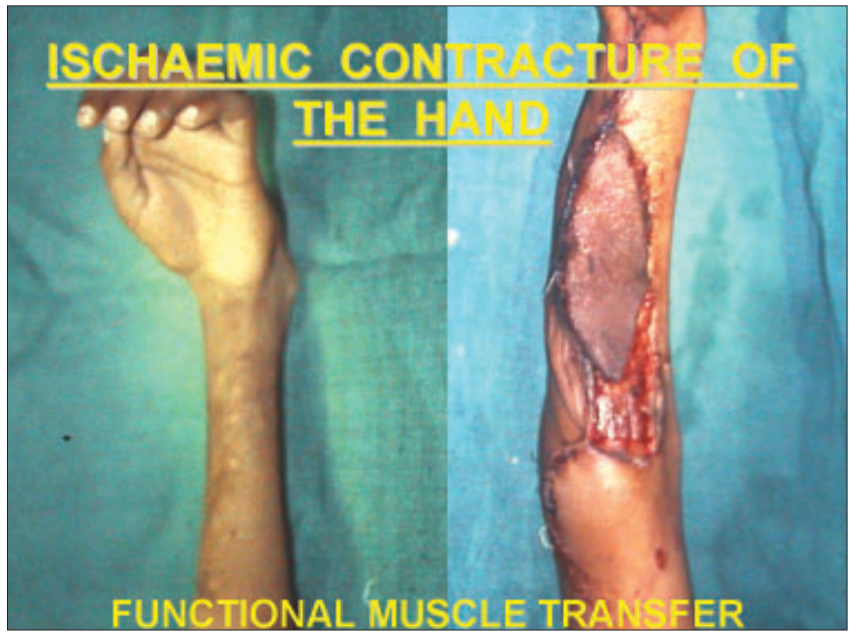

Figure 17: Functional muscle transfer

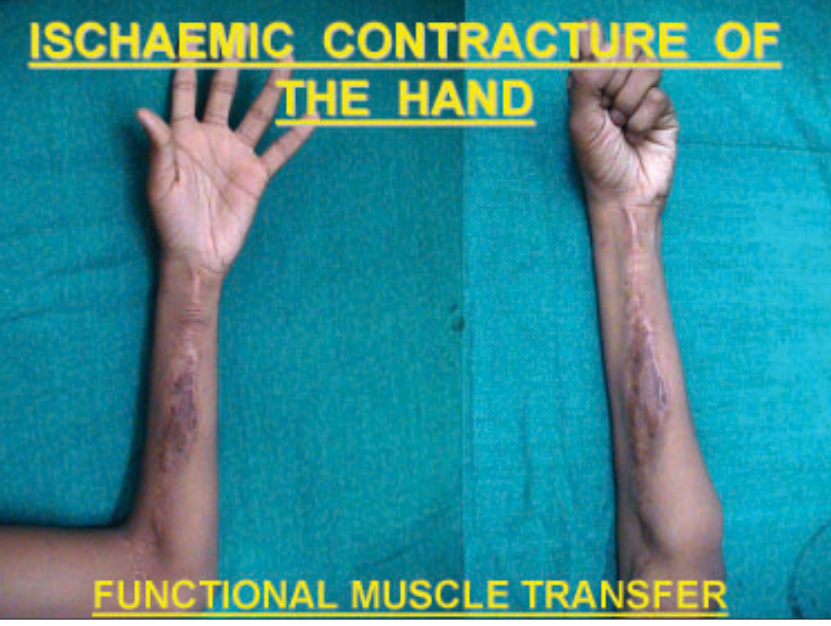

Figure 18: Functional muscle transfer

Indian J Plast Surg January-June 2006 Vol 39 Issue 1 
may be present. Paralysis of the affected muscles will be evident and there is progressing deformity.

\section{Treatment}

The goal in treatment is to re-establish the sensation, prevent stiffness, re-establish hand movements and prevent deformity.

Conservative treatment is indicated immediately after the end of acute stage, in the form of mobilization and stretching.

Surgical exploration is indicated when there is

- Altered sensation

- Failure of conservative treatment (splinting)

- CT/MRI confirmation of muscle fibrosis

Neurolysis of the median nerve along with excision of scar and the infarct is indicated when spontaneous recovery does not occur. The nerves must be displaced from the scarred flexor compartment to lie on subcutaneous tissue free from scar [Figures 5-7].

In severe lesions, nerve grafts are used to bridge the gap. Another indication for surgery at this stage is severe flexion contracture of the digits for which stretching exercises and splinting have been ineffective. During this stage, no definitive procedures such as tendon transfers should be performed. Surgeries are done only to prevent complications.

\section{Established deformity (final stage)}

As described earlier, Tsuge ${ }^{[7-9]}$ has classified deformities into the mild (localised) type, moderate (classic) type and the severe type. The various deformities that are seen include wrist flexion, Volkmann's sign [Figure 8], pronated forearm, flexed, adducted thumb, MP joints flexed and IP joints flexed / extended.

\section{Mild presentation}

In these patients only a single group of muscles, i.e. the flexors are involved and the extensors are spared. There is no neurological impairment or joint stiffness. Clinically, flexion contracture of two or three fingers, usually the middle and ring fingers is present. However, when the degree of muscle degeneration is extensive, the little and index fingers may also be affected. Sometimes, there is contracture of thumb also. A tenodesis effect may be present. A cord- like induration is felt on the flexor side. There is no involvement of the intrinsic muscles.

\section{Treatment}

Stretching [Figure 9] and regular physiotherapy will improve the condition. Tendon lengthening or transfer followed by vigorous physiotherapy may also be adequate. Where good flexor muscle mass is available by serial stretching of the flexors, we will be able to get a functional hand. The stretching should be gradual and once the full range of extension is obtained, mobilization of the fingers is essential in day time and the stretching splint may be worn in night and in between exercises. When there is loss of muscle mass, and we are able to get full straightening of the wrist and fingers, the other alternatives are tendon lengthening or tendon transfers. In the case of tendon lengthening, Z-plasty of the shortened FDS, FDP and FPL can be done or the Proximal FDS to be utilized by dividing the tendon as distally as possible and motorizing the FDP tendon by dividing it as proximally as possible [Figures 10-12].

In our experience, Z-plasties of multiple tendons' lead to significant scarring and tetherance to the skin. We do not advise such procedures now. If the extensor muscle mass is good, the best alternative is to do extensor tendon transfer to flexors.

\section{Moderate presentation}

In these patients, the flexors of the fingers, thumb and wrist are involved [Figures 13, 14]. The extensors are spared. There is however neurological (both sensory (trophic) and motor) involvement. Clinically, the classic type of Volkmann's ischaemic contracture of the hand includes flexion contracture of all fingers and thumb with wrist assuming flexed position. Claw hand deformity and trophic changes due to ulnar and median nerve involvement are also seen.

\section{Treatment}

Initial stretching followed by tendon lengthening, transfer or muscle sliding is the treatment of choice. Our preference is muscle sliding and neurolysis at the same time. Alternatively, tendon transfer can be reserved for any associated muscle fibrosis problem in the proximal forearm. Where the muscle mass is good with contractured flexors, we prefer muscle sliding [Figure 15 and 16]. While performing muscle sliding, the ulnar nerve should be protected and the entire flexor muscle 
mass along with the periosteum raised and given a new origin distally. This has worked out very well in moderate deformities. Tendon transfer also will be considered in such cases if one is not familiar with the muscle sliding procedure and if associated with the skin problem in the proximal forearm.

\section{Severe presentation}

In patients with severe presentation the flexors of the fingers, thumb and wrist are involved, and the extensors are involved partially. There is both sensory and motor neurological involvement and the skin may be involved (cicatrisation). There are often joint contractures of a severe degree and there may be associated mal-union or non-union of fracture of forearm bones.

\section{Treatment}

Surgery for the deformity is usually performed at 5-6 months. Stretching, splinting, neurolysis, bone shortening and functional muscle transfer are the options. Where there is nerve involvement, neurolysis is very important. After three months of neurolysis, the patient may be reviewed. When there is persistent contracture of the hand with no muscle mass and involvement of the extensor group, the ideal procedure is a free tissue transfer.

In free tissue transfers, we have used two musclesLattissimus dorsi and Gracilis. The advantages of Lattissimus dorsi are that it is easy to harvest and has an adequate skin component. However it is bulky, there is no tendon and invariably produces a mass action. In the case of the Gracilis, flap dissection is difficult. The viability of the skin paddle is always precarious. However, it has a long tendon, which can also be split into two to replace the deep flexors and Flexor pollicis longus. Free tissue transfer always proves to be a better alternative in severe deformities [Figures 17 and 18].

Postoperative care in these patients includes proper physiotherapy so as to have a full range of movement in the fingers and prevent wrist deformity. The patient must be immobilized for 6 weeks: 3 weeks of above elbow plaster and 3 weeks of below elbow plaster. They should also be advised to wear night splints for 3 months with regular physiotherapy.

Management of ischaemic contracture of the hand is aimed at early diagnosis and prompt decompression in the acute stage. Muscle sliding offers better results than tendon lengthening or transfer in the moderate type of contractures. In severe lesions, the procedure of choice is functional muscle transfer. The aim should be for uncomplicated recovery.

\section{Intrinsic contractures of the hand}

This condition follows intermetacarpal compartment syndrome and was first described by Finochietto in 1920 . The causes include trauma, severe persistent edema in the intermetacarpal compartments, and thermal burns to hand.

The compartments in the hand involved are the retroadductor space (the deep palmar arch begins in this space), the first intermetacarpal space and the $2^{\text {nd }}, 3^{\text {rd }}$ and $4^{\text {th }}$ intermetacarpal spaces.

The established deformities are grouped into:

Group I: All the Interosseous and Thenar muscles are involved (FPB, APB and dorsal interossei), usually associated with classic V. I. C.

Group II: Either medial thenar muscles or interosseous muscles are involved. Hence the deformity is localized to thumb or fingers.

Group III: Atypically localized to one or more fingers e.g., Abductor Digiti Quinti.

Zancolli has described the associated features as follows: Type 1: There is complete finger extension and flexion with inability to place fingers in a hook position. There is delayed flexion at the PIP joint with a positive intrinsic plus test. Mild muscular contracture can be diagnosed when there is inability to place the affected finger in a 'hook' position.

Type 2: There is Intrinsic plus deformity with MP joint flexion contracture. In this type, the Interosseous muscle contracture is greater and the IP joints are in a position of extension. There is inability to completely open the hand and the delay in flexion at the PIP joints is more accentuated. The intrinsic plus test is positive.

Type 3: In these patients there is an Intrinsic plus deformity with articular stiffness. Occasionally the affected fingers may show a swan-neck deformity. Hand function is severely affected. Thumb involvement is usually associated.

\section{Thumb deformities}

Type 1: Adduction contracture. Medial Thenar muscle 
(FPB, Adductor pollicis, Opponens) contracture presents with typical adduction contracture of the first metacarpal i.e., thumb contracted against radial aspect of index. Intrinsic plus test is positive.

Type 2: Adduction and palmar abduction contracture is seen when both medial and lateral (APB) Thenar muscles are involved. These patients present with adduction and palmar abduction contracture, blocking the flexion of index and middle fingers. The MP joint is contracted in hyper flexion i.e. intrinsic plus deformity of thumb.

\section{Treatment}

Acute stage

The swelling between the metacarpals and the pain is an indication for decompression which is done on the dorsum along the ulnar border of the second metacarpal and radial border of the fifth metacarpal. Through the second metacarpal incision, we can also release the adductor pollicis compartment.

\section{Finger deformities}

Type 1: Excision of the intrinsic lateral band and fan fibres of dorsal aponeurosis on both sides with distal intrinsic muscle release solves the problem. Post operatively, the hand is immobilised in a POP cast keeping the wrist and MP joint in a neutral position allowing IP joints to remain free for active mobilization. The cast is removed after 10 days and continued with muscle re-education. A reverse knuckle bender is useful during the re-education period. Type 2: A proximal intrinsic release is required in these patients. Release of the muscle proximal to the MP joint i.e. at musculo tendinous junctions at the level of deep transverse intermetacarpal ligament is the procedure of choice.

Type 3: A proximal intrinsic release and palmar release of MP joint at the same sitting is required. This presents as a difficult surgical situation. If there is MP joint stiffness in addition, this must be mobilized by excision of its collateral ligaments and volar plate release or in irreversible cases, the joint must be fused in the position of function.

\section{Thumb deformities}

The thumb web should be released by Z-plasty. When tissue is deficient, cover with local, regional or distant flaps is necessary. Adductor and FPB release is also mandatory.

\section{CONCLUSION}

Ischaemic contracture of the hand is a sequelae of ischaemia to muscle and nerves in a closed osteo-fascial compartment which can be best prevented by early diagnosis and fasciotomy. In our series, fifty percent of patients with Volkmann's ischemic contracture had history of fracture both bones of forearm or supracondylar fractrure, for which native treatment by traditional bonesetters was given in the form of tight bandage. This was associated with blisters in the forearm adding to the skin problem. They could not remove the tight bandage in spite of alarming pain. This warrants awareness among the members of medical profession to diagnose before it is too late and to do a prompt fasciotomy or referral. If the release is not done before the irreversible tissue damage is set in, it leads to various degrees of deformity which must be dealt with differently at different stages and for different grades of involvement to get the best possible outcome.

Finally I would like to add the adage "prevention is better than cure" Hence every one of us should spread the message to prevent this evil.

\section{REFERENCES}

1. Volkman R. Die ischaemischen Muskellahmungen und kontrakturen. Zentraibl Chir 1881;8:801-3.

2. Holden CE. Compartmental Syndrome following trauma. Clin Orthop 1975;113:95-102.

3. Mubarak SJ, Caroll NC. Volkmann's Contracture in children: Aetiology and prevention. J Bone Joint Surg 1979;61B:285-93.

4. Finochietto R. Retraccion de volkmann de los musculos intrinsicos de los manes. Bol Trab Soc Chir 1920;4:31.

5. Seddon HS. Volkmann's ischemia. Br Med J 1964;1:1587-92.

6. Zancolli E. Classification of Established Volkmann's ischemic Centratue and the program for its treatment. In: Structural and Dynamic Basis of Hand Surgery. 2nd ed. JB Lippincott: Philadephia; 1979. p. 314-24.

7. Tsuge K. Treatment of Established Volkmann's Contractune. J Bone Joint Surg 1975;57A:925-9.

8. Tsuge K. Management of Established Volkmann's contracture, In: "Green's Operative Hand Surgery". 4th ed, Churchill Livingstone: 1999. p. 592-603.

9. Tsuge K. Volkmann's Contracture. In: Comprehensive Atlas of Hand Surgery. 1st ed, Year Book Medical Publishers: 1989. p. 253-76. 